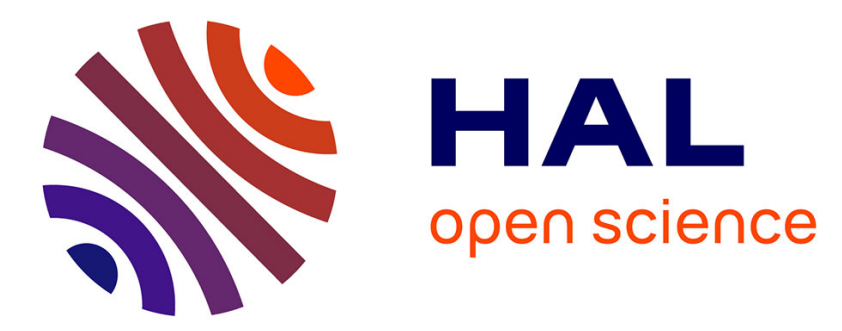

\title{
The Iso-regularization Descent Algorithm for the LASSO
}

Manuel Loth, Philippe Preux

\section{To cite this version:}

Manuel Loth, Philippe Preux. The Iso-regularization Descent Algorithm for the LASSO. 17th International Conference on Neural Information Processing, Nov 2010, Sidney, Australia. inria-00508257v2

\section{HAL Id: inria-00508257 https://hal.inria.fr/inria-00508257v2}

Submitted on 6 Sep 2010

HAL is a multi-disciplinary open access archive for the deposit and dissemination of scientific research documents, whether they are published or not. The documents may come from teaching and research institutions in France or abroad, or from public or private research centers.
L'archive ouverte pluridisciplinaire HAL, est destinée au dépôt et à la diffusion de documents scientifiques de niveau recherche, publiés ou non, émanant des établissements d'enseignement et de recherche français ou étrangers, des laboratoires publics ou privés. 


\title{
The Iso-Regularization Descent Algorithm for the LASSO
}

\author{
Manuel Loth and Philippe Preux \\ INRIA Lille - Nord Europe, \\ Université de Lille
}

\begin{abstract}
Following the introduction by Tibshirani of the LASSO technique for feature selection in regression, two algorithms were proposed by Osborne et al. for solving the associated problem. One is an homotopy method that gained popularity as the LASSO modification of the LARS algorithm. The other is a finite-step descent method that follows a path on the constraint polytope, and seems to have been largely ignored. One of the reason may be that it solves the constrained formulation of the LASSO, as opposed to the more practical regularized formulation. We give here an adaptation of this algorithm that solves the regularized problem, has a simpler formulation, and outperforms state-of-the-art algorithms in terms of speed.
\end{abstract}

Key words: Lasso, algorithm, descent, regularization

\section{Introduction}

The Least Absolute Selection and Shrinkage Operator (LASSO) was proposed in [1] as an efficient and feasible way to produce sparse linear models in regression. Let us begin by recalling its definition and fixing notations.

Let $\mathbf{v}^{\prime}, \mathbf{M}^{\prime}$ denote the transpose of a vector or matrix, $\mathbf{u}^{\prime} \mathbf{v}$ the scalar product, $\|\mathbf{v}\|_{2}^{2}=\sum_{i=1}^{\operatorname{dim}(\mathbf{v})} v_{i}^{2}=\mathbf{v}^{\prime} \mathbf{v}$ the squared $L_{2}$ norm, and $\|\mathbf{v}\|_{1}=\sum_{i=1}^{\operatorname{dim}(\mathbf{v})}\left|v_{i}\right|=$ $\operatorname{sign}(\mathbf{v})^{\prime} \mathbf{v}$ the $\ell^{1}$-norm, with $\operatorname{sign}(\mathbf{v})=\left(\operatorname{sign}\left(v_{1}\right), \ldots\right)^{\prime}$.

\section{Problem statement Given}

- $n$ samples $\left(x_{i}, y_{i}\right)_{1 \leq i \leq n}$ from correlated variables $X \in \mathcal{X}$ and $Y \in \mathbb{R}$,

- a set of feature functions $\mathcal{D} \subset \mathbb{R}^{\mathcal{X}}$,

- a constraint $t \in \mathbb{R}_{+}$,

the LASSO associates to the samples a model $Y=f(X)$ defined as a linear combination of features from $\mathcal{D}$ that minimizes the squared residual subject to an $\ell^{1}$-norm constraint on the linear coefficient vector:

$$
\min _{(\sigma, \boldsymbol{\beta}) \in 2^{\mathcal{D} \times \mathbb{R}}}\left\|\mathbf{y}-\mathbf{X}_{\sigma}{ }^{\prime} \boldsymbol{\beta}\right\|_{2}^{2} \quad \text { s.t. }\|\boldsymbol{\beta}\|_{1} \leq t,
$$

where $\sigma$, referred to as the active set, is a finite, ordered subset of features: $\sigma=\left\{\phi_{1}, \ldots, \phi_{k}\right\} \subset \mathcal{D}, \boldsymbol{\beta}$ is an associated coefficient vector, and 
$\mathbf{x}=\left[\begin{array}{c}x_{1} \\ \vdots \\ x_{n}\end{array}\right], \quad \mathbf{y}=\left[\begin{array}{c}y_{1} \\ \vdots \\ y_{n}\end{array}\right], \quad \mathbf{X}_{\sigma}=\left[\begin{array}{c}-\phi_{1}(\mathbf{x})- \\ \vdots \\ -\phi_{k}(\mathbf{x})-\end{array}\right]=\left[\begin{array}{cc}\phi_{1}\left(x_{1}\right) \ldots \phi_{1}\left(x_{n}\right) \\ \vdots & \vdots \\ \phi_{k}\left(x_{1}\right) \ldots \phi_{k}\left(x_{n}\right)\end{array}\right]$.

It should first be noted that the constraint is active only if it disqualifies all full-least-square solutions that strictly minimize the squared residual. Let $t^{*}$ be the supremum of active constraints, and let us assume in the following that $t<t^{*}$. In this case, the inequality constraint can be turned into an equality, by simple convexity arguments.

Along with defining the operator, two ways to solve the associated problem were proposed in 11. The first one is an "exterior-point" active-set method that starts from a full-least-square solution and follows a path defined by changes of active set, on which the $\ell^{1}$-norm of the coefficient decreases down to the constraint. The second one consists in casting the problem into a quadratic program (QP), and applying standard QP methods. This can be done by adding to $\mathcal{D}$ the negative counterpart of all features, thus allowing an additional nonnegativity constraint to all coefficients, which makes the $\ell^{1}$-norm constraint strictly linear.

In [2, a very different approach was introduced ("shooting" algorithm), that proceeds by means of a cyclic coordinate descent (CCD). Despite its simplicity and efficiency, it lacked popularity, partly because of the unnecessary use of the full-least-square solution as a starting point. However, it gained credit by its presentation in [3] in a more general setting.

In 4] were proposed two algorithms more related to QP, but fitted to the specificities of the problem. One is an interior-point active-set homotopy method that starts from an empty set and follows a path defined by changes of the active set on which the $\ell^{1}$-norm of the coefficient increases up to reaching the constraint. Its major interest, beside its efficiency, is that the followed path corresponds to all successive LASSO solutions for a constraint going from 0 to $t$ (regularization path). It is best known as the LASSO modification of the LARS algorithm presented in [5], in a more general framework, with more details and clarity, and in a regularized formulation. The second algorithm, that we reformulate and transpose in the following, is a surface active-set descent method: all points of the path have an $\ell^{1}$-norm equal to the constraint $t$, and the squared residual decreases down to an optimal solution.

In section 2, we give a quick yet rather detailed exposition of this method, from which we derive in section 3 an algorithm that solves the regularized formulation of the LASSO. Section 4 presents experimental results in which the new algorithm outperforms the homotopy and CCD in terms of running time. We conclude in section 5 by mentioning additional advantages of this algorithm.

\section{Iso-norm Descent}

The unnamed algorithm introduced in $\underline{4}$, that we may refer to as the iso-norm descent method, is based on the following two facts: 
- if the active set as well as the sign of the coefficients (signed active set) are known or assumed, the computation reduces to finding the minimizer of the squared residual on the corresponding hyperplane,

- the optimality of the signed active set can then easily be tested.

Let us explicit these two steps, and the algorithm that naturally arises from them.

\subsection{Minimizer on a signed active set}

If the constraint is tightened by imposing a given signed active set $(\sigma, \boldsymbol{\theta})$, where $\boldsymbol{\theta}=\operatorname{sign}(\boldsymbol{\beta})$, the problem reduces to

$$
\min _{\boldsymbol{\beta} \in \mathbb{R}^{|\sigma|}}\left\|\mathbf{y}-\mathbf{X}^{\prime} \boldsymbol{\beta}\right\|_{2}^{2} \quad \text { s.t. }\left\{\begin{array}{ll}
\boldsymbol{\theta}^{\prime} \boldsymbol{\beta} & =t \\
\operatorname{sign}(\boldsymbol{\beta}) & =\boldsymbol{\theta}
\end{array} .\right.
$$

Minimizing a convex function subject to a linear constraint is a simple QP: the minimizer is the only point on the constraint plane where the gradient of the minimized function is normal to the plane, i.e. such that any vector normal to the plane, say $\theta$, is equal to the gradient (or its negative half) up to a (Lagrange) multiplier $\lambda$ :

$$
\begin{aligned}
{[2] } & -\frac{1}{2} \nabla_{\boldsymbol{\beta}}\left\|\mathbf{y}-\mathbf{X}^{\prime} \boldsymbol{\beta}\right\|_{2}^{2}=\lambda \theta \\
& \Longrightarrow \mathbf{X}\left(\mathbf{y}-\mathbf{X}^{\prime} \boldsymbol{\beta}\right)=\lambda \boldsymbol{\theta} \\
& \Longrightarrow \mathbf{X} \mathbf{X}^{\prime} \boldsymbol{\beta}=\mathbf{X} \mathbf{y}-\lambda \boldsymbol{\theta} \\
& \Longleftrightarrow \boldsymbol{\beta}=\underbrace{\left(\mathbf{X X}^{\prime}\right)^{-1} \mathbf{X} \mathbf{y}}_{\boldsymbol{\beta}^{*}}-\lambda \underbrace{\left(\mathbf{X X}^{\prime}\right)^{-1} \boldsymbol{\theta}}_{\Delta \boldsymbol{\beta}} \\
2 & \Longleftrightarrow\left\{\begin{array}{l}
\boldsymbol{\beta}=\boldsymbol{\beta}^{*}-\lambda \Delta \boldsymbol{\beta} \\
\boldsymbol{\theta}^{\prime} \boldsymbol{\beta}=t \\
\operatorname{sign}(\boldsymbol{\beta})=\boldsymbol{\theta}
\end{array}\right. \\
& \Longleftrightarrow\left\{\begin{array}{l}
\boldsymbol{\beta}=\boldsymbol{\beta}^{*}-\lambda \Delta \boldsymbol{\beta} \\
\boldsymbol{\theta}^{\prime}\left(\boldsymbol{\beta}^{*}-\lambda \Delta \boldsymbol{\beta}\right)=t \\
\operatorname{sign}(\boldsymbol{\beta})=\boldsymbol{\theta}
\end{array}\right. \\
& \Longleftrightarrow\left\{\begin{array}{l}
\lambda=\frac{\boldsymbol{\theta}^{\prime} \boldsymbol{\beta}^{*}-t}{\boldsymbol{\theta}^{\prime} \Delta \boldsymbol{\beta}} \\
\boldsymbol{\beta}=\boldsymbol{\beta}^{*}-\lambda \Delta \boldsymbol{\beta} \\
\operatorname{sign}(\boldsymbol{\beta})=\boldsymbol{\theta}
\end{array}\right.
\end{aligned}
$$

Note that $\boldsymbol{\beta}^{*}$ is the partial least-square (PLS) solution (the least-square solution on the selected feature set), $\Delta \boldsymbol{\beta}$ can be seen as a regularization direction, and $\lambda$ as a regularization parameter.

Thus, after computing $\lambda$ and $\boldsymbol{\beta}$, it remains for this solution to satisfy the sign constraint. If it does not, this implies that $(\sigma, \boldsymbol{\theta})$ is not optimal: the squared 
residual being convex in $\boldsymbol{\beta}$, given any point $\boldsymbol{\beta}_{0}$ in the simplex (the subspace of the plane $\boldsymbol{\theta}^{\prime} \boldsymbol{\beta}$ defined by the sign constraint), it monotonically decreases on a line from $\boldsymbol{\beta}_{0}$ to $\boldsymbol{\beta}$, which will intercept the frontier of the simplex at which a coefficient (the same for any starting point $\boldsymbol{\beta}_{0}$ ) is zeroed, thus the corresponding reduced active set contains a better minimizer. The computation can then be started again on the reduced set. An example is given in Fig. 2.1 that illustrates the sign disagreement and the different variables involved.

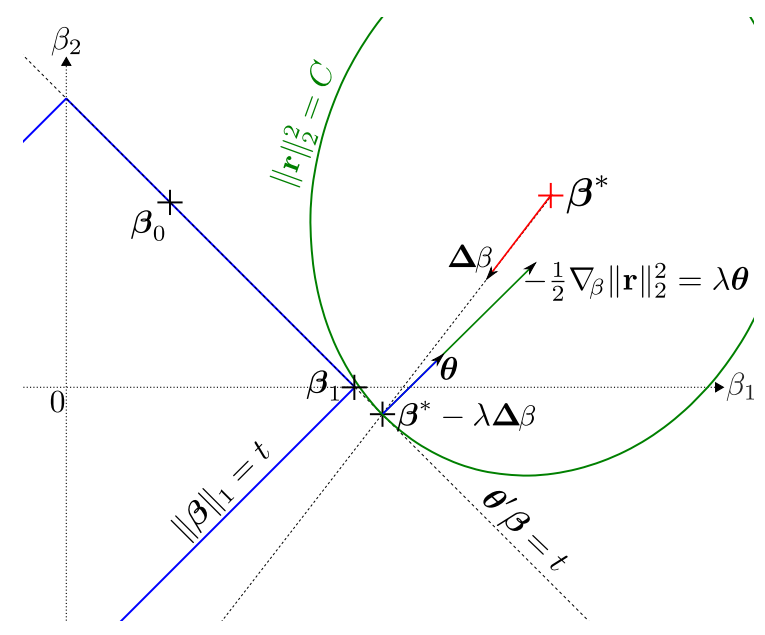

Fig. 1. From a signed active set $\left(\left\{\phi_{1}, \phi_{2}\right\},(+,+)\right)$, the regularized least-square is disagreeing with the sign hypothesis, indicating a better solution $\boldsymbol{\beta}_{1}$ on reduced active set $\left(\left\{\phi_{1}\right\},(+)\right)$. $\mathbf{r}$ is the residual $\mathbf{y}-\mathbf{X}^{\prime} \boldsymbol{\beta}$

\subsection{Karush-Kuhn-Tucker conditions}

If there is no sign disagreement, or when there is none after a sequence of optimization/shrinkage, the optimality of the active set can be tested via the necessary and sufficient Karush-Kuhn-Tucker conditions that reduce to the following $([6])$ :

$$
\left\{\begin{array}{llrl}
\forall \phi \in \sigma, & \phi(\mathbf{x})^{\prime}\left(\mathbf{y}-\mathbf{X}_{\sigma}{ }^{\prime} \boldsymbol{\beta}\right) & = & \operatorname{sign}\left(\beta_{\phi}\right) \lambda \\
\forall \phi \in \mathcal{D} \backslash \sigma, & \left|\phi(\mathbf{x})^{\prime}\left(\mathbf{y}-\mathbf{X}_{\sigma}{ }^{\prime} \boldsymbol{\beta}\right)\right| & \leq & \lambda
\end{array}\right.
$$

The first condition was handled by the previous computation, and the optimality test lies in the second one: no inactive feature should have a correlation to the residual higher than that of active features, in absolute value (over-correlation). This can be intuitively understood: the correlation being the derivative of the squared residual w.r.t. the coefficient of a feature, if an inactive feature is overcorrelated, it is possible to reduce the coefficient budget allocated to the active 
features by a sufficiently small amount and re-allocate it to the latter with greater benefice. A formal proof can be written following this sketch, that also shows that the better minimizer of the augmented active set involves an increased value of $\lambda$. Thus, given a signed active set and a sign-compliant solution to the associated (local) QP, the set augmented with an over-correlated feature contains a better solution (lower residual for equal $\ell^{1}$-norm). The sign imposed to the coefficient of the new active feature is that of its correlation, following equation 3 .

\subsection{Algorithm}

This leads to the Algorithm 1 that shrinks the active set whenever the local QP solution is not sign-compliant, otherwise expands it with the most overcorrelated feature if any, otherwise has converged to a LASSO solution. It is not mandatory to include the most over-correlated feature, but intuitively and empirically, this lessens the expected/average number of steps.

The algorithm has the descent property, since the residual decreases at each change of the active set, as shown previously, while the coefficient's $\ell^{1}$-norm is constant. It converges in at most $2^{|\mathcal{D}|}$ steps which is finite if $\mathcal{D}$ is. The number of steps is however consistently observed to be $\mathrm{O}(\min (n,|\mathcal{D}|))$ in experiments.

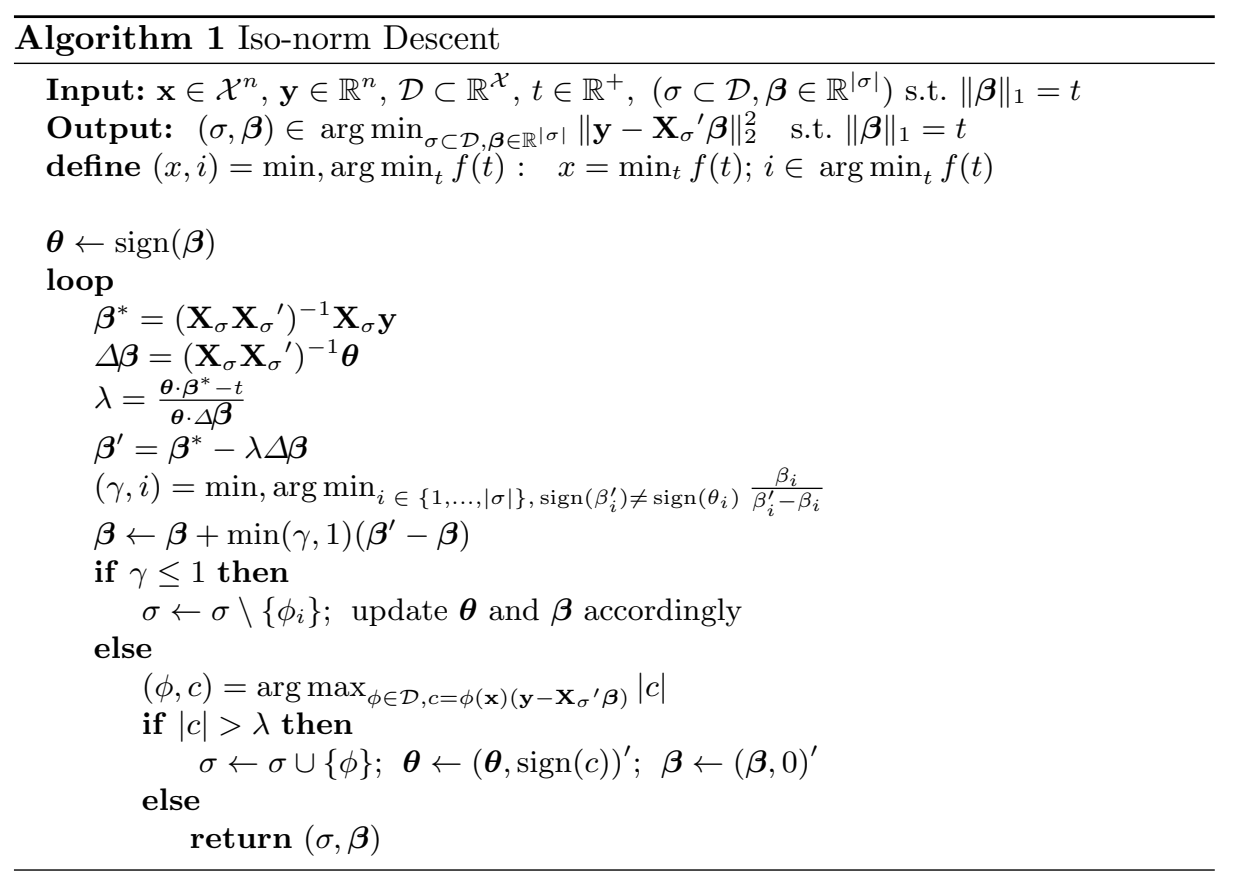




\section{Iso-Regularization Descent}

The Lagrange multiplier that appears in the resolution of the constrained leastsquare leads to an alternative regularized formulation of the LASSO: for any constraint $t$,

$$
\min _{\substack{\sigma \in 2^{\mathcal{D}} \\ \boldsymbol{\beta} \in \mathbb{R}^{|\sigma|}}}\left\|\mathbf{y}-\mathbf{X}_{\sigma}^{\prime} \boldsymbol{\beta}\right\|_{2}^{2} \quad \text { s.t. }\|\boldsymbol{\beta}\|_{1} \leq t \Longleftrightarrow \min _{\substack{\sigma \in 2^{\mathcal{D}}, \boldsymbol{\beta} \in \mathbb{R}^{|\sigma|}}} \frac{1}{2}\left\|\mathbf{y}-\mathbf{X}_{\sigma}^{\prime} \boldsymbol{\beta}\right\|_{2}^{2}+\lambda\|\boldsymbol{\beta}\|_{1}
$$

where $\lambda$ is the Lagrange multiplier associated with the minimizer(s) of the constrained formulation. It can be shown that parameters $t$ and $\lambda$ are strictly decreasing functions of each other in this equivalence relation.

For none of the formulations can the parameter easily be tuned beforehand, but $\lambda$ presents the advantage that its bounds are known in advance: the parameters such that $\mathbf{0}$ is the unique solution are $t_{\text {null }}=0$ and $\lambda \geq \lambda_{\text {null }}=$ $\max _{\phi \in \mathcal{D}}\left|\phi(\mathbf{x})^{\prime} \mathbf{y}\right|$, but the supremum $t^{*}$ of active constraints cannot be computed independently of the full-least-square solution itself, whereas the corresponding regularization parameter is $\lambda^{*}=0$. Moreover, $\lambda$ is more informative about the difference of residual between the regularized and ordinary least-square solutions.

In 7 , the authors of the iso-norm descent method mention the possibility of solving the regularized formulation by multiple runs of their algorithm inside a grid or Newton-Raphson search to find the corresponding value of $t$.

A much simpler possibility appears perhaps more clearly in our exposition of the algorithm, that consists in using a fix value of $\lambda$ - the given regularization parameter - rather than computing at each step the value maintaining a constant $\ell^{1}$-norm. This results in an even simpler algorithm described in Algorithm 2

It remains to prove that the descent property is preserved by this modification. This can be done by noting that if the $\ell^{1}$-norm of the tentative solution is lower than that of the solution, this norm is increasing in all subsequent steps, as a corollary to the fact that the Lagrange multiplier is increasing in the iso-norm descent. If we consider such a step and $\boldsymbol{\beta}_{0}$ and $\boldsymbol{\beta}_{1}$ the corresponding successive coefficients, from the convexity of the squared residual, $\boldsymbol{\beta}_{1}$ is its minimizer not only on the $\boldsymbol{\beta}^{\prime} \boldsymbol{\theta}=\boldsymbol{\beta}_{1}{ }^{\prime} \boldsymbol{\theta}$ plane, but also on the associated half-space that contains $\boldsymbol{\beta}_{0}$ and not the PLS solution. Thus any linear move toward $\boldsymbol{\beta}_{1}$ yields a monotonic improvement. It can also easily be shown that if the norm of the initial coefficient is not lower than that of the solution, the first steps the algorithm give it this property.

\section{Experiments}

We reproduced the "speed trials" experiments described in 3 with the following three algorithms: the cyclic coordinate descent algorithm described in that publication (CCD), the homotopy method, and the iso-regularization descent (iso- $\lambda$ descent). The detailed settings and source code can be found at http://chercheurs.lille.inria.fr/ loth/iso-lambda-descent_xp.tgz 


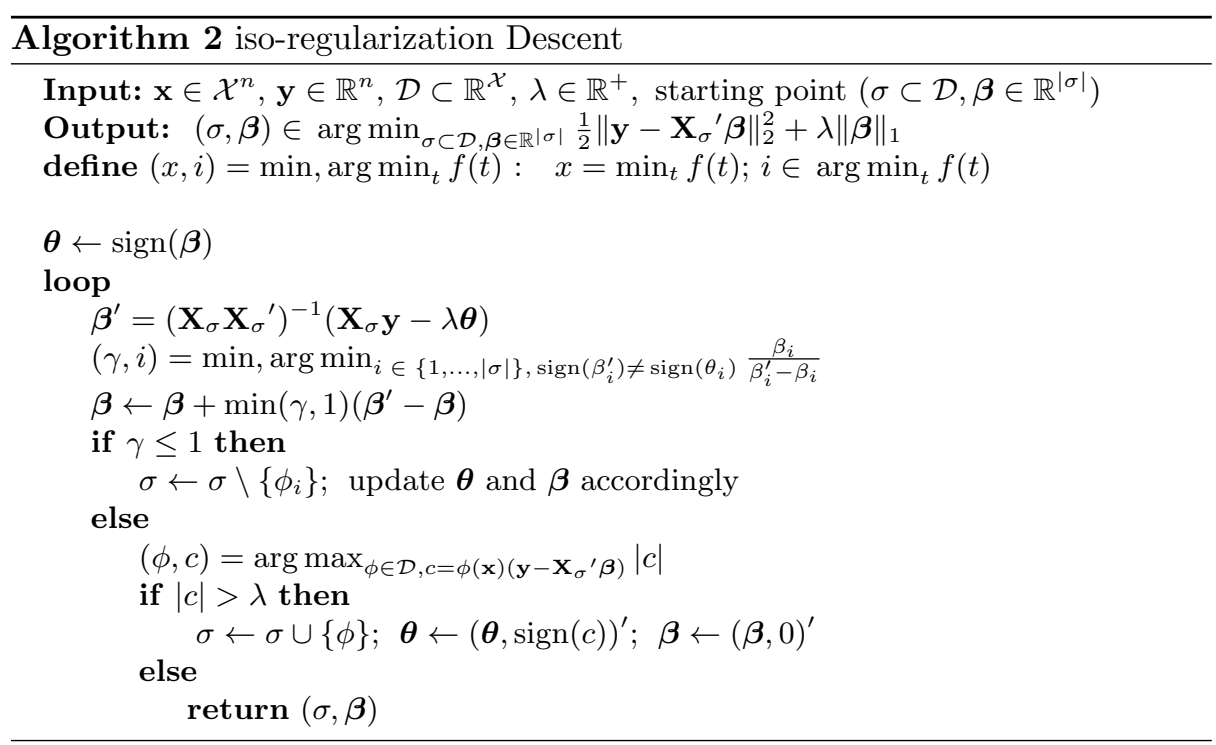

The contrast between the results of the homotopy method in our experiments and those in [3] is explained by the fact that we gave it a simplified formulation and a proper implementation in $\mathrm{C}$, closely resembling those of the iso- $\lambda$ descent. This similarity, together with a lower complexity and comparable number of steps for going from a value of $\lambda$ to another, indicate a consistently lower run time for the latter, which is confirmed by the experiments. The coordinate descent method shows slightly better results only for $|\mathcal{D}|<n$ and uncorrelated features.

\section{Conclusion}

The LASSO can thus be computed by three simple and efficient algorithms. Regardless of the running times exhibited in previous section, each offers specific advantages.

The cyclic coordinate descent is a very simple algorithm that is less subject to implementation hazards and conditioning problems. Also, since it does not involve the active Gram matrix, it supports sample reweighting and can thus be used to compute the elastic net operator. On the other hand, its convergence being asymptotic, a stopping criterion/parameter is needed.

The homotopy method gives the exact full regularization path $(\mathrm{RP})$, while keeping a computational cost of the same order as the two others. However, this is rarely needed, and solutions for a predefined set of regularization parameters are often sufficient.

In this respect, the iso-regularization descent method offers an efficient way to compute such a sequence, using one solution as a warm start for the next one, to which it converges in a small number of steps, very close to the number of 
Table 1. Speed trial experiments with the same settings as in 3. The running times, in seconds, are averaged over 10 runs. All methods and trials were implemented in $\mathrm{C}$ in similar fashions.

\begin{tabular}{|c|c|c|c|c|c|c|c|c|}
\hline \multirow[t]{2}{*}{$n$} & \multirow[t]{2}{*}{$|\mathcal{D}|$} & \multirow[t]{2}{*}{ Method } & \multicolumn{6}{|c|}{ Population correlation between features } \\
\hline & & & 0 & 0.1 & 0.2 & 0.5 & 0.9 & 0.95 \\
\hline \multirow[t]{9}{*}{100} & \multirow[t]{3}{*}{1000} & \multirow{3}{*}{$\begin{array}{l}\text { homotopy } \\
\text { iso- } \lambda \text { descent } \\
\text { CCD }\end{array}$} & 0.13 & 0.12 & 0.13 & 0.14 & 0.14 & 0.14 \\
\hline & & & 0.09 & 0.09 & 0.09 & 0.10 & 0.10 & 0.10 \\
\hline & & & 0.21 & 0.21 & 0.24 & 0.46 & 1.21 & 2.64 \\
\hline & \multirow[t]{3}{*}{5000} & \multirow{3}{*}{$\begin{array}{l}\text { homotopy } \\
\text { iso- } \lambda \text { descent } \\
\text { CCD }\end{array}$} & 0.63 & 0.65 & 0.62 & 0.67 & 0.62 & 0.60 \\
\hline & & & 0.53 & 0.56 & 0.54 & 0.59 & 0.54 & 0.54 \\
\hline & & & 1.39 & 1.39 & 1.54 & 2.32 & 7.39 & 8.53 \\
\hline & \multirow[t]{3}{*}{20000} & \multirow{3}{*}{$\begin{array}{l}\text { homotopy } \\
\text { iso- } \lambda \text { descent } \\
\text { CCD }\end{array}$} & 2.39 & 3.06 & 2.69 & 3.22 & 3.30 & 3.36 \\
\hline & & & 2.06 & 2.56 & 2.25 & 2.68 & 2.76 & 2.85 \\
\hline & & & 5.38 & 7.22 & 6.06 & 11.14 & 37.24 & 47.07 \\
\hline \multirow[t]{3}{*}{1000} & \multirow[t]{6}{*}{100} & "homotopy & 0.22 & 0.22 & 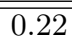 & $\overline{0.22}$ & 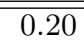 & 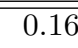 \\
\hline & & iso- $\lambda \mathrm{d}$ & 0.19 & 0.19 & 0.19 & 0.19 & 0.17 & 0.13 \\
\hline & & CCD & 0.18 & 0.23 & 0.30 & 0.65 & 1.62 & 2.13 \\
\hline \multirow[t]{3}{*}{5000} & & homotopy & 0.71 & 0.70 & 0.70 & 0.70 & 0.70 & 1.05 \\
\hline & & iso- $\lambda$ descent & 0.67 & 0.67 & 0.67 & 0.66 & 0.65 & 0.97 \\
\hline & & CCD & 0.61 & 0.75 & 0.91 & 1.44 & 4.31 & 8.07 \\
\hline
\end{tabular}

steps on the RP. The path followed between the two is itself close to the RP, as it shares the property of simultaneously increasing the coefficient $\ell^{1}$-norm and decreasing the squared residual. Another interesting property of this algorithm is its ability to handle continuous feature sets, for example the set of all Gaussian functions over $\mathcal{X}=\mathbb{R}^{p}$, parameterized by their covariance matrix. This will be investigated, together with expositions of the proofs mentioned here, in a publication to come.

\section{References}

1. Tibshirani, R.: Regression shrinkage and selection via the lasso. Journal of the Royal Statistical Society. Series B (Methodological) 58(1) (1996) 267-288

2. Fu, W.J.: Penalized Regressions: The Bridge versus the Lasso. Journal of Computational and Graphical Statistics 7(3) (September 1998) 397

3. Friedman, J., Hastie, T., Höfling, H., Tibshirani, R.: Pathwise coordinate optimization. Annals of Applied Statistics 1(2) (2007) 302-332

4. Osborne, M., Presnell, B., Turlach, B.: A new approach to variable selection in least squares problems. IMA Journal of Numerical Analysis 20(3) (2000) 389

5. Efron, B., Hastie, T., Johnstone, I., Tibshirani, R.: Least angle regression. The Annals of Statistics 32(2) (April 2004) 407-499

6. Nash, S.G., Sofer, A.: Linear and nonlinear programming. McGraw-Hill (New York) (1996)

7. Osborne, M., Presnell, B., Turlach, B.A.: On the LASSO and Its Dual. Journal of Computational and Graphical Statistics 9(2) (2000) 319 - 337 